\title{
A Recursive Resource Allocation Algorithm for MIMO-OFDMA Systems in Multi-user Context
}

\author{
Wafa BEN HASSEN, Mériem AFIF and Sami TABBANE \\ Mediatron: Research Unit on Radio Communication and Multimedia Networks, \\ Higher School of Communication of Tunis (Sup'com), \\ Carthage University, \\ Tunis, Tunisia
}

\begin{abstract}
This paper presents a new resource allocation algorithm for downlink multi-user MIMO-OFDMA systems. The main objective is to maximize the total system capacity and to guarantee the fairness among users with a low complexity. The proposed algorithm is based on a recursive sub-carriers allocation procedure aiming to satisfy Quality of Service (QoS) requirements for each mobile user. Simulation results demonstrate that the proposed algorithm performs well in terms of system capacity and fairness when the number of users in the cell is important closing to the practical case.
\end{abstract}

\section{General Terms}

4G technologies, wireless communication, optimization problem, Radio Resource Management (RRM).

\section{Keywords}

OFDMA, MIMO, sub-carriers allocation, capacity, fairness, computational complexity.

\section{INTRODUCTION}

In wireless packet access networks, increasing demands for data services with high rate requirements have imposed serious challenges on system design and link budgets [2]. Orthogonal Frequency Division Multiplexing (OFDM) is a crucial modulation technique to fulfil these requirements thanks to its ability to combat multipath fading, Inter-Symbol Interferences (ISI), and time-varying injurious effects in wireless environments [1],[7]. Moreover, Multiple Input Multiple Output (MIMO) is another promising technology that permits to achieve spectral efficiency as it employs multiple antennas at both the transmitter and the receiver to exploit spatial diversity [5]. Therefore, MIMO-OFDMA is considered as the most efficient method to achieve a high performance transmission in advanced wireless networks [11], [12].

Resources can be considered as components of a 3 dimensional object where sub-carriers, Time-Slots (TS), and spatial layers correspond to frequency, time, and space resources, respectively [10]. In the Downlink (DL) sense, the Base Station (BS) decides which resource to be allocated to which Mobile Station (MS) based on Channel State Information (CSI) feedbacks. An exponential number of allocation ways is possible even for a small number of resources and users. Therefore, resource allocation optimization problems are under intensive investigation, aiming at either maximizing the total system capacity under power constraints, called Rate Adaptive (RA) problems, or minimizing the totalpower under rate constraints, referred to as Margin Adaptive (MA) problems. There is also another type that aims to achieve the highest possible throughput by maintaining fairness among active users.

In Multi-User (MU) MIMO-OFDM systems, resource allocation problems depend on: (1) Spatial Division Multiple Access (SDMA) grouping problem. (2) Pre-coding transmitting signals problem. (3) Power allocation problem. (4) Sub-carrier assignment problem. Optimal resource allocation strategy must jointly solve the above-mentioned sub-problems. It leads, generally, to a complex combinatorial and non-convex optimization problem and then considered as a Non-deterministic Polynomial-time Hard (NPHard) problem [4]. For these reasons, recent researches propose a suboptimal method in order to close optimal solution with a low complexity. The common oneis to solve above sub-problems separately, called here "distributed" resource allocation problem.

In this paper, a sub-optimal resource allocation problem in a downlink multi-user MIMO-OFDM system is proposed. The main objective is to maximize the total system capacity and to guarantee the fairness among users with a low complexity. The proposed method includes different step keys: (1) Determine dominant eigen-channels. (2) Sort sub-carriers in decreasing order for each user. (3) Sort users in decreasing order depending on their dominant eigen-channels. (4) Remaining sub-carriers allocation. The particularity of this algorithm relies on its recursive sub-carriers allocation procedure aiming to satisfy Quality of Service (QoS) requirements for each mobile user.

The remainder of this paper is described as follows. In Section 2, related works in OFDM-MIMO systems are well-discussed. Then, system model is presented and optimization problem is formulated in section 3 . In section 4 , the recursive sub-carrier allocation algorithm is explained in details. Finally, simulation results and performance analysis are introduced to compare the proposed method with another existing one.

\section{RELATED WORKS}

In packet access networks, Radio Resource Management (RRM) for downlink MIMO-OFDM systems has attracted enormous research interests. Here, sub-optimal resource allocation strategies are modelled by Fig.1 [10].

In this section, a literature review on sub-optimal resource allocation strategies is provided [3],[4],[9],[13]. In [4], the Spatial Division Multiple Access (SDMA) grouping, resource assignment and power allocation problems are jointly solved 
as a convex optimization problem. In order to reduce complexity, authorsconsider two special cases which are: user selection and antennasselection. Zero Forcing (ZF) pre-coding

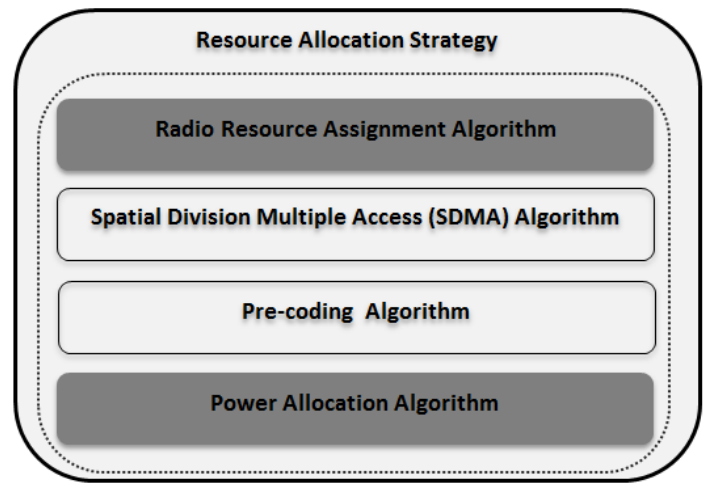

Fig 1: Sub-optimal Resource Allocation Strategies in MIMO-OFDM Systems

is employed. After that, the proposed algorithms are extended to develop a sub-optimal algorithm for the optimal optimization problem which allows the sub-space selection. In [9], authors aim to maximize the total capacity subject to constraints on total available power, Bit Error Rate (BER) and rate proportions. A distributed resource allocation method is proposed: (1) Sub-carrier allocation is based on grouped eigen-channels. Once the dominant eigen-channel is determined to be allocated to user $k$, the corresponding subcarrier is also allocated to this user. (2) Two power allocation methods are investigated in this paper, one is equal power allocation for each sub-carrier and the other is the sub-optimal power allocation.

In [3], dynamic resource allocation problems are investigated, aiming to minimize the total transmit power depending on the fact that Quality of Service (QoS) of different users can be guaranteed such as BER. Optimal power allocation is obtained thanks to the greedy water-filling algorithm application. Then, in order to minimize the total transmit power of the whole system, a specified sub-carrier should be allocated to user $k$ whose required transmit power is minimum over sub-carrier $n$. After sub-carrier allocation is completed, adaptive bit allocation scheme is applied. Here, a set of available spatial sub-channels for each user is divided into clusters or chunks. The average CSI within each cluster is used to replace the CSI of each spatial sub-channel within acluster. Then, data bits for different users can be allocated to their spatial sub-channels based on clusters using a greedy water-filling algorithm.

In [13], a Rate Adaptive (RA) optimization problem subject to an additional proportional fairness constraint is developed. Here, the optimization problem is divided into two steps; the first step is to decide which sub-carriers are allocated to each user based on its dominant eigen-channels. The second step is the power allocation scheme that depends on the previous sub-carriers allocation step. The power of the first user in its first sub-carrier is calculated based on the total power constraint. Then, the power of each sub-carrier is obtained through linear relations.

In this paper, a sub-optimal resource allocation problem in MU MIMO-OFDM systems is proposed. The main objective is to maximize the total system capacity and to guarantee fairness among users with a low complexity. Here, the power is equally distributed among users and sub-carriers in order to reduce complexity. Hence, authors are interested, in this paper, by the sub-carriers allocation scheme in order to fulfil the main goal.

\section{SYSTEM MODEL AND PROBLEM FORMULATION}

Fig. 2 shows the block diagram of a downlink MU MIMO-OFDMA system.

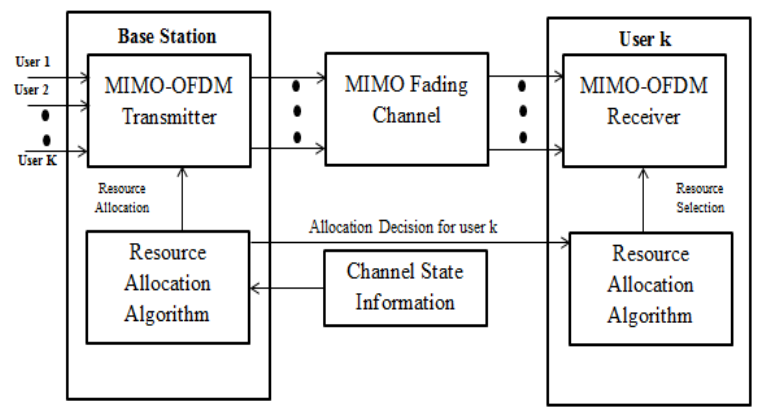

Fig 2: Downlink MIMO-OFDMA System Diagram

In this paper, a MIMO-OFDMA system that consists of a single BS and $K$ users randomlydistributed in a single cell. B represents the total bandwidth divided into Nsub-carriers. The number of transmit antennas and receive antennas are $N_{t}$ $\operatorname{and} N_{r}$, respectively. For each user $k$ on sub-carriernis assigned a power $p_{k, n}$ and a channel state matrix $G_{k, n}$ with size $\left(N_{r} \times N_{t}\right)$.

$G_{k, n}$ is decomposed through Singular Value Decomposition (SVD) as follows:

$$
G_{k, n}=U_{k, n} \Sigma_{k, n} V_{k, n}=\sum_{i=1}^{r} u_{k, n}^{i} \lambda_{k, n}^{i}\left(v_{k, n}^{i}\right)^{H}
$$

Assuming that the eigenvalues of $G_{k, n} G_{k, n}^{H}$ are $\left\{\lambda_{k, n}^{i}\right\}_{i=1}^{r}$ where $r=\min \left(N_{r}, N_{t}\right)$ is the rank of $G_{k, n}$ and $\left\{u_{k, n}^{i}\right\}_{i=1}^{r}$ and $\left\{v_{k, n}^{i}\right\}_{i=1}^{r}$ are the corresponding singular vectors.

The maximum singular value $\lambda_{k, n}^{\wedge}$ is generally much larger than the others where $\lambda_{k, n}^{\wedge}=\max \left\{\lambda_{k, n}^{i}\right\}_{i=1}^{r}$. It is used referred to as the dominant eigen-channels for user $k$ on sub-carrier $n$. Note that the received Signal to Noise Ratio (SNR) $H_{k, n}^{i}$ is defined as:

$$
H_{k, n}^{i}=\frac{\left(\lambda_{k, n}^{i}\right)^{2}}{N_{0}\left(\frac{B}{N}\right) \Gamma}
$$

where $\Gamma$ is a constant SNR gap which is related with the required BER, expressed as [14]:

$$
\Gamma=\frac{\operatorname{Ln}(-5 B E R)}{1.5}
$$

Then, the data rate of user $k$ is defined as follows:

$$
R_{k}=\frac{B}{N} \sum_{n=1}^{N^{k}} \sum_{i=1}^{r} \log _{2}\left(1+p_{k, n} H_{k, n}^{i}\right)
$$

where $N^{k}$ denotes the number of sub-carriers allocated to user $k$ and $p_{k, n}$ is the power allocated to user $k$ on sub-carrier $n$ with: 
where $P_{T O T}$ is the total transmitting power by the BS.

$$
p_{k, n}=\frac{P_{T O T}}{N}(5)
$$

The following assumptions are used in the proposed algorithm:

- All users in the system require the same service.

- Each sub-carrier may be allocated to only one user at any given time.

- Power is equally distributed among sub-carriers.

- $\quad$ Each user has a perfect Channel State Information (CSI) back to the base stationthrough a feedback channel.

Having the goal to maximize the system capacity, the optimization problem is formulatedas follows:

\section{Maximize}

$$
C_{s y s}=\frac{B}{N} \sum_{k=1}^{K} \sum_{n=1}^{N^{k}} \sum_{i=1}^{r} \log _{2}\left(1+p_{k, n} H_{k, n}^{i}\right)(6)
$$

Subject to

$$
\begin{aligned}
& \sum_{k=1}^{K} N^{k}=N(7 . \mathrm{a}) \\
& \sum_{k=1}^{K} \sum_{n=1}^{N^{k}} p_{k, n}=P_{T O T} \\
& p_{k, n} \geq 0, \forall k, n
\end{aligned}
$$

\section{A RECURSIVE SUB-CARRIER ALLOCATION ALGORITHM}

In this section, a new algorithm aiming to maximize the system capacity fairness is well described and its computational complexity is evaluated. Based on MIMO CSI, dominant eigen-channels with gains in (2) are used to sort sub-carriers in decreasing order. Then, a recursive step is applied to allocate sub-carriers.Here, users are sorted in decreasing order according to the matrix of sorted subcarriers $H_{k, n}^{\prime}$. In this case, the matrix of sorted users $\mathrm{H}_{\mathrm{k}, \mathrm{n}}^{\prime \prime}$ is obtained. Then, each user is assigned its best sub-carrier. However, if the sub-carrier is allocated to a more prior user, the next free and best sub-carrier is searched and the data rate in (4) is updated. After allocating to each user its best subcarrier, the first column from the matrix of sorted user $H_{k, n}^{\prime \prime}$ is truncated and the matrix $H_{k, n}^{\prime}$ is obtained. The same recursive step is applied for the remaining sub-carriers until the number of sub-carriers required by each user $N_{k}$ is satisfied. Finally, remaining sub-carriers allocation step is applied until the number of free sub-carriers is equal to zero which permits to enhance the system capacity.

\section{BEGIN}

(I)

$$
\begin{aligned}
& \text { Initialization } \\
& \Lambda=\{1,2, \ldots, \mathrm{K}\} \\
& \Gamma=\{1,2, \ldots, N\} \\
& r=\min \left(N_{t}, N_{r}\right) \\
& R_{k}=0, \forall k \in \Lambda ; \\
& p=P_{T O T} / N ; \\
& \Omega_{k}=\phi ; \\
& N_{k}=\gamma N
\end{aligned}
$$

(II) Dominant Eigen-Channels Determination

$$
\text { for } k=1 \text { to } K \text { do }
$$

for $\mathrm{n}=1$ toNdo

$\lambda_{k, n}^{\wedge} \leftarrow$ Find dominant eigenvalue with the maximum eigen-

$$
\text { channels value }\left\{\lambda_{k, n}^{i}\right\}_{i=1}^{i=r} \text {. }
$$

end for

end for

\section{(III) Required Sub-carriers Ordering}

for $k=1$ to $K$ do

$H_{k, n}^{\prime} \leftarrow$ Order sub-carriers according to its best dominant eigenvalue $\lambda_{k, n}^{\wedge}$.

end for

(IV) Sub-carriers Allocation

while $\Gamma \neq \phi$ or $N_{1 \rightarrow K}=0$ do

$\mathrm{j}=1$;

$H_{k, n}^{\prime \prime} \leftarrow$ Order users in decreasing order according to $H_{k, n}^{\prime}$.

for $k=1$ to $K$ where $\operatorname{order}(k)<\operatorname{order}(k+1)$ do

$$
\text { if } N_{k}=0 \text { then }
$$

$k=k+1 \%$ move to the next user

else

if sub-carrier $j$ is not allocated then

$$
\begin{aligned}
& \Gamma \leftarrow \Gamma-\{j\} ; \\
& \Lambda \leftarrow \Lambda-\{k\} ;
\end{aligned}
$$$$
\Omega_{k}=\Omega_{k} \cup\{\mathrm{j}\} ; N_{k}=N_{k}-1
$$

$\%$ allocate sub-carrier $j$ to user $k$

else

Update data rate $R_{k}$ according to equation (4).

$\%$ sub-carrier is allocated to more prior user

Repeat

$j=j+1 \%$ search the next free and best sub-carrier

untilFind a free sub-carrierj

end if

end if

end for

$H_{k, n}^{\prime} \leftarrow$ Truncate the first column from the matrix $\mathrm{H}_{\mathrm{k}, \mathrm{n}}^{\prime \prime}$ end while

Reset $\Lambda=\{1,2, \ldots, K\}$

(V) Remaining Sub-carriers Allocation

while $\Gamma \neq \phi$

$\mathrm{j}=1$;

$H_{k, n}^{\prime \prime} \leftarrow$ Order users in decreasing order according to $H_{k, n}^{\prime}$.

fork $=1$ to $K$ where $\operatorname{order}(k)<\operatorname{order}(k+1)$ do

if sub-carrier $j$ is not allocated then

$$
\Gamma \leftarrow \Gamma-\{j\} ; \Lambda \leftarrow \Lambda-\{k\}
$$

$\Omega_{k}=\Omega_{k} \cup\{\mathrm{j}\} ; \%$ allocate sub-carrier $j$ to user $k$

else

Update data rate $R_{k}$ according to equation (4).

$\%$ sub-carrier is allocated to more prior user

Repeat

$j=j+1 \%$ search the next free and best sub-carrier

untilfind a free sub-carrier $j$

end if

end for

$H_{k, n}^{\prime} \leftarrow$ Truncate the first column from the matrix $H_{k, n}^{\prime \prime}$

end while

Return $R_{k}$ and $\Omega_{k}$

END

\section{Computational Complexity:}

Let us recall that $K$ and $N$ refer to the users and sub-carriers number, respectively. Step (III) sorts sub-carriers in descending order for each user which requires $K N \log _{2}(N)$. Then, in step (IV), users are sorted $\left(N-N^{\prime}\right)$ times, then requires $\left(N-N^{\prime}\right) K \log _{2}(K)$ operations where $N^{\prime}$ is the number of remaining sub-carriers. Finally, in step (V), remaining sub-carriers are allocated to sorted users, so $\left(N^{\prime} K \log _{2}(K)+K N^{\prime} \log _{2}\left(N^{\prime}\right)\right)$ operations are required. Thus, the asymptotic complexity is $O\left(N K\left(\log _{2}(N K)\right)\right.$.

\section{SIMULATION RESULTS}

In this work, the channel is modeled as a Rayleigh Channel with four multi-paths. The simulated system consists of a 
single cell that uses 1024 sub-carriers for communications. In order to consider channel state variation, the channel state matrix changes every sub-frame duration and the simulation window is equal to 10000 sub-frames. Gaussian white noise variance $N_{0}$ is 0.5 and BER is $10^{-3}$.It is assumed that all users require the same service such as File Transfer Protocol (FTP) service. Moreover, each user require at least 16 sub-carriers to reach its required throughput. Simulation parameters are described in Table 1.

\section{Table 1. Simulation Parameters}

\begin{tabular}{|l|c|c|}
\hline \multicolumn{1}{|c|}{ Parameter } & Symbol & Value \\
\hline Total Bandwidth $(\mathrm{MHz})$ & $\mathrm{B}$ & 10 \\
\hline Number of sub-carriers & $N$ & 1024 \\
\hline Number of transmitter antennas & $N_{t}$ & 4 \\
\hline Number of receiver antennas & $N_{r}$ & 4 \\
\hline Sub-frame delay $(\mu \mathrm{s})$ & $T_{S F}$ & 714,286 \\
\hline Sub-carriers spacing $(\mathrm{KHz})$ & $\Delta F$ & 7.813 \\
\hline
\end{tabular}

The proposed resource allocation algorithm is compared with a sub-optimal existing solution, called Zhao's algorithm [14]. The proposed and Zhao's algorithm have the same optimization problem aim. Zhao\&al [14] formulates the problem of maximizing thetotal system capacity with proportional rate constraints in a MIMO-OFDMA system. It uses the sub-carrierswith high SNR for each user to achieve a better system capacity.Moreover, it introduces a proportional fairness procedure in order to satisfy fairness among users with a low complexity.

Fig. 3 shows the total spectral efficiency among the proposed algorithm Zhao's algorithm, the number of users varies from 1 to 50 with an interval 5 .

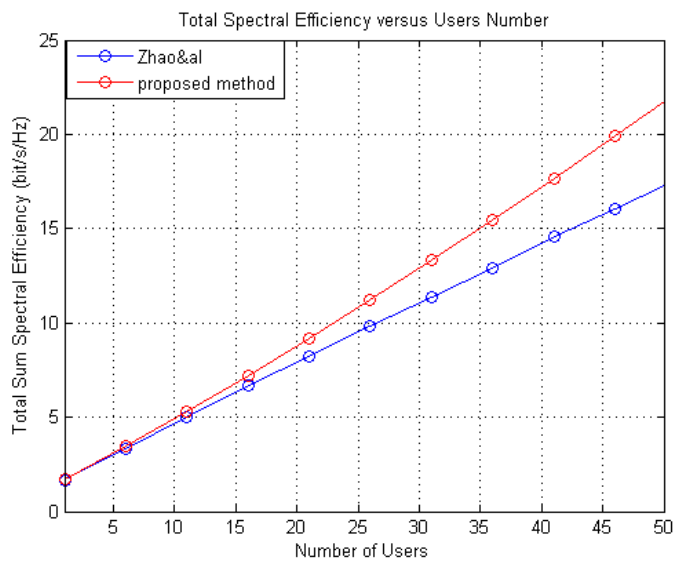

Fig 3: Total Sum Spectral Efficiency versus Users' Number
Table2. Variation Intervals in Terms of Spectral Efficiency

\begin{tabular}{|c|c|c|c|c|c|}
\hline & $\begin{array}{l}10 \\
10 \\
0\end{array}$ & $\begin{array}{l}{\left[\begin{array}{lll}1 & 0 & \\
2 & 0 & {[}\end{array}\right]}\end{array}$ & $\begin{array}{ll}{\left[\begin{array}{ll}2 & 0 \\
3 & 0\end{array}\right]}\end{array}$ & $\begin{array}{l}{\left[\begin{array}{ll}3 & 0 \\
4 & 0\end{array}\right]}\end{array}$ & $\begin{array}{l}{\left[\begin{array}{ll}4 & 0 \\
5 & 0\end{array}\right]} \\
]\end{array}$ \\
\hline$S E_{P Z I}$ & $\begin{array}{l}0.0 \\
83\end{array}$ & $\begin{array}{l}0.47 \\
4\end{array}$ & $\begin{array}{l}1.14 \\
2\end{array}$ & $\begin{array}{l}2.14 \\
2\end{array}$ & $\begin{array}{l}3.49 \\
9\end{array}$ \\
\hline
\end{tabular}

Table 2 shows variation intervals in terms of total sum spectral efficiency. Let $S E_{P Z H}$ denote the total Spectral Efficiency in different variation users' intervals. These values are computed based onthe mean difference between the contribution and Zhao\&al method described in [14].

As $S E_{P Z H}>0$, it is obvious that the proposed method provides greater spectral efficiency than the Zhao\&al method [14]. The difference between theproposed contribution and the existing method increases when the number of users rises, meaning that the proposed method operates well with multiusers diversity, especially when the number of users is important, closing to the practical case.This is explained by the fact that the proposed scheme searches for the next free and best sub-carriers, when the best sub-carriers for user $k$ isyet allocated to a more prior user.

Fig.4 shows Jain Fairness Index (JFI) among proposed algorithm and Zhao\&al algorithm that proposes to use proportional rates to guarantee fairness among users.

The Jain Fairness Index is computed as [8]:

$$
J_{\text {Fair Index }}=\frac{\left(\sum_{k=1}^{K} R_{k, \text { mean }}\right)^{2}}{K \times\left(\sum_{k=1}^{K}\left(R_{k, \text { mean }}\right)^{2}\right)}
$$

where $R_{k, \text { mean }}$ is the mean data rate of user $k$ in a simulation time. This indicator is given by the following formula [6]:

$$
R_{k, \text { mean }}=\frac{1}{b} \sum_{i=1}^{b=T / T_{S F}} R_{k, i}
$$

where $R_{k, i}$ is the data rate of mobile station $k$ in sub-frame $i$.

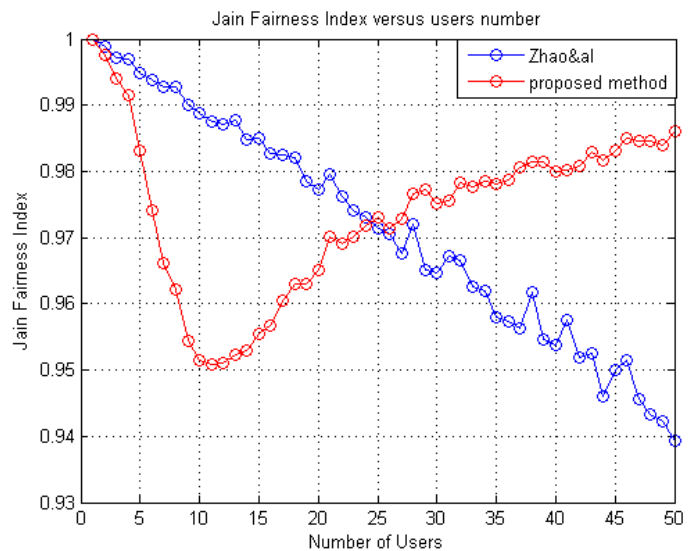

Fig 4: Jain Fairness Index versus Users' Number 
Table 3. Variation Intervals in Terms of Jain Fairness Index

\begin{tabular}{|c|c|c|c|c|c|}
\hline & $\begin{array}{l}10, \\
10[\end{array}$ & $\begin{array}{lll}{\left[\begin{array}{lll}1 & 0 & 0 \\
2 & 0 & {[}\end{array}\right]} & \end{array}$ & $\begin{array}{ll}{\left[\begin{array}{lll}2 & 0 \\
3 & 0 & {[}\end{array}\right.}\end{array}$ & $\begin{array}{l}{\left[\begin{array}{ll}3 & 0, \\
4 & 0\end{array}\right]}\end{array}$ & $\begin{array}{l}{\left[\begin{array}{ll}4 & 0 \\
5 & 0\end{array}\right]} \\
\text {, }\end{array}$ \\
\hline$J F_{P Z H}$ & $\begin{array}{l}- \\
0.0 \\
1\end{array}$ & $\begin{array}{ll}- & \\
0.02 \\
8\end{array}$ & $\begin{array}{l}- \\
9.7 \mathrm{e} \\
-4\end{array}$ & $\begin{array}{l}0.01 \\
7\end{array}$ & $\begin{array}{l}0.03 \\
3\end{array}$ \\
\hline
\end{tabular}

Table 3 shows variation intervals in terms of Jain Fairness Index. Let $J F_{P Z H}$ denote the Jain Fairness Index in different variation users' intervals. These values are computed based onthe mean difference between theproposed contribution and Zhao\&al method described in [14].

When the number of users is lower than 25 , it is obvious that the proposed method provides lower fairness than the proportional fairness method [14] as $J F_{P Z H}<0$. However, when the number of users is important, $k>25$, the proposed method guarantees greater fairness, meaning that the proposed method provides a better tradeoff between system capacity and fairness when the number of users in the cell is important,because the proportional fairness constraint in [14] is inefficient when the number of users is important: $R_{1}: R_{2}: \ldots: R_{K}=\theta_{1}: \theta_{2}: \ldots: \theta_{K}$ as $\theta_{i}$ depends on the number of users in the system. Note that the algorithm Zhao\&al[14] is simulatedonly for 8 users which explains its inefficiency when the number of users is more important, $k>25$.

Fig.5, a twice vertical bar graph, compares two sets of data mean spectral efficiency per user. One bar represents the proposed method and the other one represents Zhao\&al method [14].

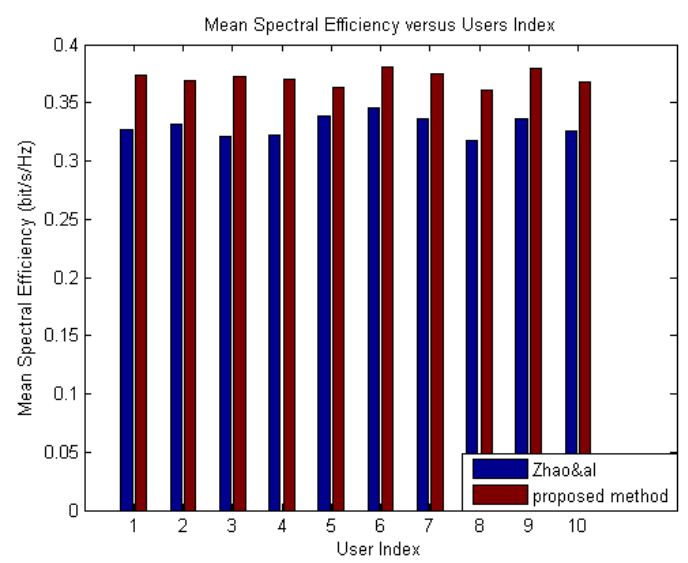

Fig 5: Mean Spectral Efficiency versus Users' Index

It is clear that the proposed method provides better mean spectral efficiency per user than the other existing method [14]. Moreover, it is obvious that the spectral efficiency provided to each user by the proposed method is almost the same, which is not the case for proportional fairness algorithm [14]. Notice that the number of sub-carriers required for each user is equal to 16 sub-carriers.

Simulation results demonstrate that the proposed scheme permits to achieve a better trade-off between fairness and system capacity compared to another recent method Zaho\&al [14] as it employs a recursive step for sub-carriers allocation. Moreover, the proposed algorithm presents a low complexity and then may be adopted for real-time applications as it allocates resource (sub-carrier and power) separately.

\section{CONCLUSION}

This paper has considered the problem of resource allocation for mobile users in context of MIMO-OFDMA system. It aims to resolve the problem of sub-carriers assignment to mobile users, in order to maximize the total system capacity. In this context, a new dynamic algorithm consisting of different steps which are dominant eigen-channels determination, sub-carriers sorting for each user and users sorting, has been proposed and compared with an alternative previously proposed in the literature [14]. The proposed scheme uses a recursive procedure for sub-carriers allocation. Numerical results show that the proposed algorithm permits to achieve a better trade-off between fairness and efficiency use of resources compared to another existing method. In addition to this contribution, the new algorithm presents a low complexity and may be adopted for real-time applications. As future works, this work may be extended to a multi-cell multiservice context.

\section{REFERENCES}

[1] 3GPP, "Physical Layer Aspects for Evolved Universal Terrestrial Radio Access (UTRA)," Technical Report TR.25.814, V.7.1.0, 3rd. Generation Partnership Project, Sep 2006.http://www.3gpp.org

[2] F. Akyildiz and and Elias Chavarria Reyes David M. Gutierrez-Estevez. "The Evolution to $4 \mathrm{G}$ Cellular Systems: LTE-Advanced". Physical Communications, 3:217-244,2010.

[3] D. Bin, C.C. Ko, and Y. Liang. "An Enhanced Capacity and Fairness Scheme for MIMO-OFDMA Downlink Resource Allocation". In ISCIT '07: International Symposium on Communications and Information Technologies, pages 495-499, Oct 2007.

[4] P. W. C. Chan and R. S. Cheng. "Capacity maximization for Zero-Forcing MIMO-OFDMA Downlink Systems with Multiuser Diversity". IEEE Transactions on Wireless Communications, 6(5):1880-1889, May 2007.

[5] M. Doettling, M. Sternad, G. Klang, J. Von Hafen, and M. Olsson. "Integration of Spatial Processing in the WINNER B3G Air Interface Design". In VTC '06: Proceedings of the IEEE 63rd Vehicular Technology Conference, volume 1, pages 246-250, 2006.

[6] W. B. Hassen and M. Afif. "A Gain-Computation Enhancements Resource Allocation for Heterogeneous Service Flows in IEEE $802.16 \mathrm{~m}$ Mobile Networks". International Journal of Digital Multimedia Broadcasting, 2012:1-13, 2012.

[7] IEEE. "Air interface for fixed broadband wireless access systems”. Technical Report IEEE 802.16-2004, Institute of Electrical and Electronics Engineers, Oct 2004.

[8] R. Jain, D.M. Chiu, and W. Hawe. "A Quantitative Measure of Fairness And Discrimination for Resource Allocation in Shared Computer Systems". CoRR, 1998.

[9] K. B. Letaief and Y. J. Zhang. "Dynamic Multiuser Resource Allocation and Adaptation for Wireless Systems". IEEE Transactions on Wireless Communications, 13(4):38-47, Aug 2006. 
[10] T.F. Maciel and A. Klein. "On the Performance, Complexity and Fairness of Suboptimal Resource Allocation for Multi-User MIMO-OFDMA Systems". IEEE Transactions on Vehicular Technology, 59:406419, Jan 2010.

[11] D.W.K. Ng, E.S. Lo, and R. Schober. "Dynamic Resource Allocation in MIMO-OFDMA Systems with Full-Duplex and Hybrid Relaying”. IEEE Transactions on Communications, 60(5):1291-1304, May 2012.

[12] Pratibha, Jagannatham, and Aditya K. "Power allocation for MIMO-OFDM based CR with spatial constraints and CSI uncertainty". In SAM '12: 7th IEEE Sensor Array and Multichannel Signal Processing Workshop, pages 137-140, June 2012.

[13] S. Qiaoyun, T. Hui, D. Kun, W. Shengdong, and Z. Ping. "A Novel Resource Allocation Algorithm for Multiuser Downlink MIMO-OFDMA". In WCNC '08: IEEE Wireless Communications and Networking Conference, pages 1844-1848, March-April 2008.

[14] J. Zhao and Y. Li. "An Efficient Proportional Resource Allocation Algorithm in MIMO-OFDMA Systems". In WiCOM'11: International Conference on Wireless Communications, Networking and Mobile Computing, pages $\quad 1-4, \quad$ Sep 2011 . 